\title{
Representation of odd integers as the sum of one prime, two squares of primes and powers of 2
}

\author{
by \\ TAO LiU (Jinan)
}

1. Introduction. In 1999, Liu-Liu-Zhan [6, Theorem 4] proved that every large odd $N$ can be represented as the sum of one prime, two squares of primes and $k$ powers of 2 . Let $r_{k}(N)$ denote the number of solutions of the equation

$$
N=p_{1}+p_{2}^{2}+p_{3}^{2}+2^{\nu_{1}}+2^{\nu_{2}}+\cdots+2^{\nu_{k}} .
$$

One may anticipate that a small $k$ in (1.1) is not sufficient to give the positivity of $r_{k}(N)$. Since it is well known that one of the fundamental problem on the solubility of additive equations is to determine a lower bound for the number of variables in the equation, an interesting question arises of how many powers of 2 are needed to ensure $r_{k}(N)>0$.

In this paper, we shall show that $k \geq 22000$, so a not very large number of variables in the equation (1.1) is sufficient to ensure $r_{k}(N)>0$. More precisely, we have the following result:

TheOREM 1. Let $r_{k}(N)$ be as defined above. Then there exists a constant $k_{0} \geq 22000$ and a constant $N_{k}$ depending on $k$ only such that if $N \geq N_{k}$, $k \geq k_{0}$, then $r_{k}(N)>0$.

2. The circle method. First, we give some notations. Define

$$
P=N^{2 / 15-\varepsilon}, \quad Q=N / P L^{14}, \quad L=\log N .
$$

For $\alpha \in[1 / Q, 1+1 / Q]$, from Dirichlet's lemma on rational approximation, we have

$$
\alpha=a / q+\lambda, \quad|\lambda| \leq 1 / q Q
$$

where $1 \leq a \leq q \leq Q,(a, q)=1$. Take

$$
M(a, q)=[a / q-1 / q Q, a / q+1 / q Q],
$$

2000 Mathematics Subject Classification: 11Pxx. 


$$
\begin{gathered}
\mathbf{M}=\bigcup_{q \leq P} \bigcup_{a=1,(a, q)=1}^{q} M(a, q), \\
C(\mathbf{M})=[1 / Q, 1+1 / Q] / \mathbf{M} .
\end{gathered}
$$

Since $2 P \leq Q$, the sets $M(a, q)$ are mutually disjoint.

For $\chi \bmod q$, define

$$
\begin{array}{ll}
\mathrm{C}(\chi, a)=\sum_{h=1}^{q} \bar{\chi}(h) e\left(\frac{a h^{2}}{q}\right), & \mathrm{C}\left(\chi^{0}, a\right)=C(a, q), \\
\mathrm{D}(\chi, a)=\sum_{h=1}^{q} \bar{\chi}(h) e\left(\frac{a h}{q}\right), & \mathrm{D}\left(\chi^{0}, a\right)=D(a, q) .
\end{array}
$$

If $\chi_{1}, \chi_{2}, \chi_{3}$ are characters $\bmod q$, we define

$$
\begin{aligned}
B\left(n, q, \chi_{1}, \chi_{2}, \chi_{3}\right) & =\sum_{a=1,(a, q)=1}^{q} e\left(-\frac{a n}{q}\right) \mathrm{C}\left(\chi_{1}, a\right) \mathrm{C}\left(\chi_{2}, a\right) \mathrm{D}\left(\chi_{3}, a\right), \\
B(n, q) & =B\left(n, q, \chi_{1}^{0}, \chi_{2}^{0}, \chi_{3}^{0}\right), \\
\sigma_{1}(n) & =\sum_{q \leq P} \frac{B(n, q)}{\phi^{3}(q)} .
\end{aligned}
$$

Define

$$
\begin{aligned}
T_{1}(\alpha) & =\sum_{p \leq N} \log p e(\alpha p), & S_{1}(\alpha) & =\sum_{p^{2} \leq N} \log p e\left(\alpha p^{2}\right), \\
T(\alpha) & =\sum_{M<p \leq N} \log p e(\alpha p), & S(\alpha) & =\sum_{M<p^{2} \leq N} \log p e\left(\alpha p^{2}\right),
\end{aligned}
$$

where $M=N L^{-14}$.

Theorem 1 depends mainly on Theorem 2:

Theorem 2. For $N / 2 \leq n \leq N$, we have

$$
\int_{\mathbf{M}} S_{1}^{2}(\alpha) T_{1}(\alpha) e(-n \alpha) d \alpha=\frac{\pi}{4} \sigma_{1}(n) n+O\left(N L^{-1}\right) .
$$

LEMMA 2.1. Let $\chi_{j}, j=1,2,3$, be primitive characters $\bmod r_{j}, r_{0}=$ $\left[r_{1}, r_{2}, r_{3}\right]$, and $\chi^{0}$ a principal character mod $q$. Then for any $\varepsilon>0$ there exists $c(\varepsilon)$ such that

$$
\sum_{q \leq x, r_{0} \mid q} \frac{1}{\phi^{3}(q)}\left|B\left(n, q, \chi_{1} \chi^{0}, \chi_{2} \chi^{0}, \chi_{3} \chi^{0}\right)\right| \leq_{c(\varepsilon)} r_{0}^{-1 / 2+\varepsilon} L^{10} .
$$

Proof. The proof is similar to that of Lemma 7 of [2], so we leave it to the reader as an exercise. Define 
(2.2) $W(\chi, \lambda)=\sum_{M<p^{2} \leq N} \log p \chi(p) e\left(p^{2} \lambda\right)-\delta_{\chi} \sum_{M<m^{2} \leq N} e\left(m^{2} \lambda\right)$,

$$
U(\chi, \lambda)=\sum_{M<p \leq N} \log p \chi(p) e(\alpha p)-\delta_{\chi} \sum_{M<m \leq N} e(m \lambda),
$$

where

$$
\delta_{\chi}= \begin{cases}1 & \text { if } \chi=\chi^{0} \\ 0 & \text { if } \chi \neq \chi^{0}\end{cases}
$$

Obviously, we have

$$
\begin{aligned}
& S(\alpha)=\frac{C(a, q)}{\phi(q)} V_{1}(\lambda)+\frac{1}{\phi(q)} \sum_{\chi \bmod q} \mathrm{C}(\chi, a) W(\chi, \lambda), \\
& T(\alpha)=\frac{D(a, q)}{\phi(q)} V_{2}(\lambda)+\frac{1}{\phi(q)} \sum_{\chi \bmod q} \mathrm{D}(\chi, a) U(\chi, \lambda) .
\end{aligned}
$$

Then

$$
\int_{\mathbf{M}} S^{2}(\alpha) T(\alpha) e(-n \alpha) d \alpha=I_{1}+I_{2}+2 I_{3}+I_{4}+2 I_{5}+I_{6},
$$

where

$$
\begin{aligned}
I_{1}= & \sum_{q \leq P} \frac{\mu(q)}{\phi^{3}(q)} \sum_{a=1,(a, q)=1}^{q} e\left(-\frac{a n}{q}\right) C^{2}(a, q) \\
& \times \int_{-1 / q Q}^{1 / q Q}\left(\sum_{M \leq m^{2} \leq N} e\left(m^{2} \lambda\right) m^{-1}\right)^{2}\left(\sum_{M \leq m \leq N} e(m \lambda)\right) e(-n \lambda) d \lambda, \\
I_{2}= & \sum_{q \leq P} \frac{1}{\phi^{3}(q)} \sum_{a=1,(a, q)=1}^{q} \mu(q) e\left(-\frac{a n}{q}\right) \\
& \times \int_{-1 / q Q}^{1 / q Q} V_{2}(\lambda)\left(\sum_{\chi \bmod q} \mathrm{C}(\chi, a) W(\chi, \lambda)\right)^{2} e(-n \lambda) d \lambda, \\
I_{3}= & \sum_{q \leq P} \frac{1}{\phi^{3}(q)} \sum_{a=1,(a, q)=1}^{q} \mu(q) C(a, q) e\left(-\frac{a n}{q}\right) \\
& \times \int_{-1 / q Q}^{1 / q Q} V_{1}(\lambda) V_{2}(\lambda)\left(\sum_{\chi \bmod q} \mathrm{C}(\chi, a) W(\chi, \lambda)\right) e(-n \lambda) d \lambda,
\end{aligned}
$$




$$
\begin{aligned}
I_{4}= & \sum_{q \leq P} \frac{1}{\phi^{3}(q)} \sum_{a=1,(a, q)=1}^{q} C^{2}(a, q) e\left(-\frac{a n}{q}\right) \\
& \times \int_{-1 / q Q}^{1 / q Q} V_{1}^{2}(\lambda)\left(\sum_{\chi \bmod q} \mathrm{D}(\chi, a) U(\chi, \lambda)\right) e(-n \lambda) d \lambda \\
I_{5}= & \sum_{q \leq P} \frac{1}{\phi^{3}(q)} \sum_{a=1,(a, q)=1}^{q} C(a, q) e\left(-\frac{a n}{q}\right) \\
& \times \int_{-1 / q Q}^{1 / q Q} V_{1}(\lambda)\left(\sum_{\chi \bmod q} \mathrm{C}(\chi, a) W(\chi, \lambda)\right)\left(\sum_{\chi \bmod q} \mathrm{D}(\chi, a) U(\chi, \lambda)\right) e(-n \lambda) d \lambda, \\
I_{6}= & \sum_{q \leq P} \frac{1}{\phi^{3}(q)} \sum_{a=1,(a, q)=1} e\left(-\frac{a n}{q}\right) \\
& \times \int_{-1 / q Q}^{1 / q Q}\left(\sum_{\chi \bmod q} \mathrm{C}(\chi, a) W(\chi, \lambda)\right)^{2}\left(\sum_{\chi \bmod q} \mathrm{D}(\chi, a) U(\chi, \lambda)\right) e(-n \lambda) d \lambda .
\end{aligned}
$$

We also define

$$
\begin{gathered}
J^{\prime}=\sum_{r_{1} \leq P} r_{1}^{-1 / 4+\varepsilon} \sum_{\chi_{1} \bmod r_{1}}^{*} \max _{|\lambda| \leq 1 / r_{1} Q}\left|W\left(\chi_{1}, \lambda\right)\right| \\
K=\sum_{r_{2} \leq P} r_{2}^{-1 / 4+\varepsilon} \sum_{\chi_{2} \bmod r_{2}}^{*}\left(\int_{-1 / r_{2} Q}^{1 / r_{2} Q}\left|W\left(\chi_{2}, \lambda\right)\right|^{2} d \lambda\right)^{1 / 2}, \\
J=\sum_{r_{3} \leq P} r_{3}^{-\varepsilon} \sum_{\chi_{3} \bmod r_{3}}^{*} \max _{|\lambda| \leq 1 / r_{3} Q}\left|U\left(\chi_{3}, \lambda\right)\right|, \\
K_{1}=\left(\int_{-1 / Q}^{1 / Q}\left|V_{2}(\lambda)\right|^{2} d \lambda\right)^{1 / 2}, \quad K_{2}=\left(\int_{-1 / Q}^{1 / Q}\left|V_{1}(\lambda)\right|^{2} d \lambda\right)^{1 / 2} .
\end{gathered}
$$

First, we estimate $I_{6}$ :

$$
\begin{aligned}
\left|I_{6}\right|= & \mid \sum_{q \leq P} \frac{1}{\phi^{3}(q)} \sum_{\chi_{1} \bmod q} \ldots \sum_{\chi_{3} \bmod q} B\left(n, q, \chi_{1}, \chi_{2}, \chi_{3}\right) \\
& \times \int_{-1 / q Q}^{1 / q Q} W\left(\chi_{1}, \lambda\right) W\left(\chi_{2}, \lambda\right) U\left(\chi_{3}, \lambda\right) e(-n \lambda) d \lambda \mid \\
\leq & \sum_{r_{1} \leq P} \ldots \sum_{r_{3} \leq P} \sum_{\chi_{1} \bmod r_{1}}^{*} \ldots \sum_{\chi_{3} \bmod r_{3}}^{*} \sum_{q \leq P, r_{0} \mid q}\left|\frac{B\left(n, q, \chi_{1} \chi^{0}, \chi_{2} \chi^{0}, \chi_{3} \chi^{0}\right)}{\phi^{3}(q)}\right| \\
& \times \int_{-1 / q Q}^{1 / q Q}\left|W\left(\chi_{1} \chi^{0}, \lambda\right) W\left(\chi_{2} \chi^{0}, \lambda\right) W\left(\chi_{3} \chi^{0}, \lambda\right)\right| d \lambda,
\end{aligned}
$$


where $\chi^{0}$ is a principal character $\bmod q, r_{0}=\left[r_{1}, r_{2}, r_{3}\right]$, and the notation $\sum^{*}$ refers to primitive characters. For $q \leq P$ and $M \leq p \leq N$, we have $(q, p)=1$. Using this and (2.2), for the primitive character $\chi_{3}$, we get $W\left(\chi_{3} \chi^{0}, \lambda\right)=W\left(\chi_{3}, \lambda\right)$. Thus by Lemma 2.1 we obtain

$$
\begin{aligned}
\left|I_{6}\right| \leq & \sum_{r_{1} \leq P} \cdots \sum_{r_{3} \leq P} \sum_{\chi_{1} \bmod r_{1}}^{*} \ldots \sum_{\chi_{3} \bmod r_{3}}^{*} \\
& \times \int_{-1 / r_{0} Q}^{1 / r_{0} Q}\left|W\left(\chi_{1}, \lambda\right)\right|\left|W\left(\chi_{2}, \lambda\right)\right|\left|W\left(\chi_{3}, \lambda\right)\right| d \lambda \\
& \times \sum_{q \leq P, r_{0} \mid q}\left|\frac{B\left(n, q, \chi_{1} \chi^{0}, \chi_{2} \chi^{0}, \chi_{3} \chi^{0}\right)}{\phi^{3}(q)}\right| \\
\ll & L^{10} \sum_{r_{1} \leq P} \sum_{r_{2} \leq P} \sum_{r_{3} \leq P} r_{0}^{-1 / 2+\varepsilon} \sum_{\chi_{1} \bmod r_{1}}^{*} \sum_{\chi_{2} \bmod r_{2}}^{*} \sum_{\chi_{3} \bmod r_{3}}^{*} \\
& \times \int_{-1 / r_{0} Q}\left|W\left(\chi_{1}, \lambda\right)\right|\left|W\left(\chi_{2}, \lambda\right)\right|\left|U\left(\chi_{3}, \lambda\right)\right| d \lambda \\
\ll & L^{10} \sum_{r_{3} \leq P} r_{3}^{-\varepsilon} \sum_{\chi_{3} \bmod r_{3}}^{*} \max _{\mid \leq 1 / r_{3} Q}\left|U\left(\chi_{3}, \lambda\right)\right| \\
& \times \sum_{r_{1} \leq P} r_{1}^{-1 / 4+\varepsilon} \sum_{\chi_{1} \bmod r_{1}}^{*}\left(\int_{-1 / r_{1} Q}^{1 / r_{1} Q}\left|W\left(\chi_{1}, \lambda\right)\right|^{2} d \lambda\right)^{1 / 2} \\
& \times \sum_{r_{2} \leq P} r_{2}^{-1 / 4+\varepsilon} \sum_{\chi_{2} \bmod r_{2}}^{*}\left(\int_{-1 / r_{2} Q}^{1 / r_{2} Q}\left|W\left(\chi_{2}, \lambda\right)\right|^{2} d \lambda\right)^{1 / 2} \\
= & L^{10} J K^{2} . \quad
\end{aligned}
$$

For $I_{5}$, we have the following estimate:

$$
\begin{aligned}
\left|I_{5}\right| \leq & L^{10} \sum_{r_{2} \leq P} \sum_{r_{3} \leq P}\left[r_{2}, r_{3}\right]^{-1 / 2+\varepsilon} \sum_{\chi_{2} \bmod r_{2}}^{*} \sum_{\chi_{3} \bmod r_{3}}^{*} \\
& \times \int_{-1 / r_{0} Q}^{1 / r_{0} Q}\left|W\left(\chi_{2}, \lambda\right)\right|\left|V_{1}(\lambda)\right|\left|U\left(\chi_{3}, \lambda\right)\right| d \lambda \\
\leq & L^{10} \sum_{r_{3} \leq P} r_{3}^{-\varepsilon} \sum_{\chi_{3} \bmod r_{3}}^{*} \max _{|\lambda| \leq 1 / r_{3} Q}\left|U\left(\chi_{3}, \lambda\right)\right|\left(\int_{-1 / Q}^{1 / Q}\left|V_{1}(\lambda)\right|^{2} d \lambda\right)^{1 / 2} \\
& \times \sum_{r_{2} \leq P} r_{2}^{-1 / 4+\varepsilon} \sum_{\chi_{2} \bmod r_{2}}^{*}\left(\int_{-1 / r_{2} Q}^{1 / r_{2} Q}\left|W\left(\chi_{2}, \lambda\right)\right|^{2} d \lambda\right)^{1 / 2} \\
= & L^{10} J K K_{2} .
\end{aligned}
$$


Similarly, we have

$$
\begin{aligned}
\left|I_{4}\right| \leq & L^{10} \sum_{r_{3} \leq P} r_{3}^{-\varepsilon} \sum_{\chi_{3} \bmod r_{3}}^{*} \max _{|\lambda| \leq 1 / r_{3} Q}\left|U\left(\chi_{3}, \lambda\right)\right| \\
& \times\left(\int_{-1 / Q}^{1 / Q}\left|V_{1}(\lambda)\right|^{2} d \lambda\right)^{1 / 2}\left(\int_{-1 / Q}^{1 / Q}\left|V_{1}(\lambda)\right|^{2} d \lambda\right)^{1 / 2} \\
= & L^{10} J K_{2}^{2}, \\
\left|I_{3}\right| \ll & L^{10} \sum_{r_{2} \leq P} r_{2}^{-1 / 4+\varepsilon} \sum_{\chi_{2} \bmod r_{2}}^{*} \max _{|\lambda| \leq 1 / r_{2} Q}\left|W\left(\chi_{2}, \lambda\right)\right| \\
& \times\left(\int_{-1 / Q}^{1 / Q}\left|V_{1}(\lambda)\right|^{2} d \lambda\right)^{1 / 2}\left(\int_{-1 / Q}^{1 / Q}\left|V_{2}(\lambda)\right|^{2} d \lambda\right)^{1 / 2} \\
= & L^{10} J^{\prime} K_{1} K_{2}, \sum_{r_{1} \leq P} r_{1}^{-1 / 4+\varepsilon} \sum_{\chi_{1} \bmod r_{1}}^{*} \max _{|\lambda| \leq 1 / r_{1} Q}\left|W\left(\chi_{1}, \lambda\right)\right| \\
& \times \sum_{I_{2} \leq P} r_{2}^{-1 / 4+\varepsilon} \sum_{\chi_{2} \bmod r_{2}}^{*}\left(\int_{-1 / r_{2} Q}^{1 / r_{2} Q}\left|W\left(\chi_{2}, \lambda\right)\right|^{2} d \lambda\right)^{1 / 2}\left(\int_{-1 / Q}^{1 / Q}\left|V_{2}(\lambda)\right|^{2} d \lambda\right)^{1 / 2} \\
= & L^{10} J^{\prime} K K_{1} . \quad \sum^{1 / 2}
\end{aligned}
$$

Now, we estimate $J^{\prime}, J, K, K_{1}$ and $K_{2}$. Since

$$
V_{2}(\lambda)=\sum_{M<m \leq N} e(m \lambda) \ll \min (N, 1 /|\lambda|),
$$

we have

$$
K_{1} \leq\left(\int_{0}^{1 / N} N^{2} d \lambda+\int_{1 / N}^{\infty} \lambda^{-2} d \lambda\right)^{1 / 2} \ll \sqrt{N}
$$

Since

$$
\begin{aligned}
V_{1}(\lambda) & =\sum_{M<m^{2} \leq N} e\left(m^{2} \lambda\right)=\int_{\sqrt{M}}^{\sqrt{N}} e\left(\lambda u^{2}\right) d u+O(1+|\lambda| N) \\
& =\frac{1}{2} \sum_{M<m \leq N} m^{-1 / 2} e(m \lambda)+O(1+|\lambda| N),
\end{aligned}
$$

we obviously have

$$
V_{1}(\lambda) \ll \min \left(\sqrt{N}, \frac{1}{\sqrt{M}}|\lambda|^{-1}\right)+O(|\lambda| N+1) .
$$


Thus

$$
\left|V_{1}(\lambda)\right|^{2} \ll\left(\min \left(\sqrt{N}, \frac{1}{\sqrt{M}}|\lambda|^{-1}\right)\right)^{2}+O\left(1+|\lambda|^{2} N^{2}\right) .
$$

Next,

$$
\begin{aligned}
K_{2} & =\left(\int_{-1 / Q}^{1 / Q}\left|V_{1}(\lambda)\right|^{2} d \lambda\right)^{1 / 2} \\
& \ll\left(\int_{-1 / Q}^{1 / Q} \min \left(N, \frac{1}{M}|\lambda|^{-2}\right) d \lambda\right)^{1 / 2}+\left(\int_{-1 / Q}^{1 / Q}\left(1+|\lambda|^{2} N^{2}\right) d \lambda\right)^{1 / 2} .
\end{aligned}
$$

The first term on the right hand side is

$$
\ll\left(\int_{0}^{1 / \sqrt{M N}} N d \lambda+\int_{1 / \sqrt{M N}}^{\infty} \frac{1}{M} \lambda^{-2} d \lambda\right)^{1 / 2} \ll\left(L^{7}+L^{7}\right)^{1 / 2} \ll L^{4},
$$

while the second is $\ll 1$. Therefore,

$$
K_{2} \ll L^{4} .
$$

For $J^{\prime}$ and $K$, we have the following lemmas:

Lemma 2.2 (see [4, Lemma 5.1]). Let $A>0$ be arbitrary. Then

$$
J^{\prime} \ll N^{1 / 2} L^{-A}
$$

where the $O$-constant depends at most on $A$.

LEMma 2.3 (see [4, Lemma 6.1]). We have

$$
K \ll L^{c},
$$

where $c>0$ is an absolute constant.

Now it remains to estimate $J$. This is similar to Lemma 5.1 of [4]. For completeness, we write it out in detail.

Lemma 2.4. Let $A>0$ be arbitrary. Then there exists a constant $B=$ $B(A)>0$ such that

$$
J \ll N L^{-A}
$$

where the $O$-constant depends at most on $A$.

Proof. Since

$$
J \ll \max _{R \leq P} J_{R} L
$$

where the definition of $J_{R}$ is similar to that of $J$ except that the sum is over $r \sim R$, we only need to prove $J_{R} \ll N L^{-A}$. We divide the proof into two cases: $L^{B}<R \leq P$ and $R \leq L^{B}$. So we need to prove the following three lemmas. 
LEMMA 2.5. We have

$$
\sum_{r \sim R} \sum_{\chi \bmod r}^{*} \max _{|\lambda| \leq 1 / r Q}|U(\chi, \lambda)| \ll R^{\varepsilon} N L^{-A}
$$

Let

$$
\widehat{U}(\chi, \lambda)=\sum_{M<m \leq N}\left(\Lambda(m) \chi(m)-\delta_{\chi}\right) e(m \lambda)
$$

Then

$$
U(\chi, \lambda)-\widehat{U}(\chi, \lambda)=-\sum_{j \geq 2} \sum_{M<p^{j} \leq N} \log p \chi(p) e\left(p^{j} \lambda\right) \ll N^{1 / 2},
$$

hence (2.5) becomes a consequence of the following lemma:

LEMMA 2.5'. We have

$$
\sum_{r \sim R} \sum_{\chi \bmod r}^{*} \max _{|\lambda| \leq 1 / r Q}|\widehat{U}(\chi, \lambda)| \ll R^{\varepsilon} N L^{-A}
$$

where $L^{B} \leq R \leq P$ and $A$ is a positive number.

This is proved by a slight modification of the proof of Lemma 5.1 of [4] (replace $N^{1 / 2}$ by $N$ ).

For $R \leq L^{B}$, we have the following result:

Lemma 2.6. Let $A>0, B>0$ be arbitrary numbers. Then for $R \leq L^{B}$, we have $J_{R} \ll N L^{-A}$, where the implied constant depends at most on $A$ and $B$.

The proof is similar to that of Lemma 5.5 of [4] (replace $T=N^{1 / 6}$ by $\left.T=N^{1 / 3}\right)$.

Now, we can estimate $I_{1}, \ldots, I_{6}$. We have

$$
\begin{aligned}
& \left|I_{6}\right| \ll L^{10} J K^{2} \ll L^{10} N L^{2 c} L^{-A} \ll N L^{-A}, \\
& \left|I_{5}\right| \ll L^{10} J K K_{2} \ll L^{10} N L^{-A} L^{4+c} \ll N L^{-A}, \\
& \left|I_{4}\right|=L^{10} J K_{2}^{2} \ll L^{10} N L^{-A} L^{8}, \\
& \left|I_{3}\right| \ll L^{10} J^{\prime} K_{1} K_{2} \ll L^{10} N^{1 / 2} L^{-A} N^{1 / 2} L^{4} \ll N L^{-A}, \\
& \left|I_{2}\right| \ll L^{10} J^{\prime} K K_{1} \ll L^{10} N L^{-A} L^{c} \ll N L^{-A} .
\end{aligned}
$$

Thus, we have

$$
\int_{\mathbf{M}} T(\alpha) S^{2}(\alpha) e(-n \alpha) d \alpha=I_{1}+I_{2}+2 I_{3}+I_{4}+2 I_{5}+I_{6}=I_{1}+O\left(N L^{-A}\right) \text {. }
$$

It remains to deal with $I_{1}$. From (2.3) and (2.4), we have

(*) $I_{1}=\frac{1}{4} \sum_{q \leq P} \frac{\mu(q)}{\phi^{3}(q)} \sum_{a=1,(a, q)=1}^{q} e\left(-\frac{a n}{q}\right) C^{2}(a, q)$ 


$$
\begin{aligned}
& \times \int_{-1 / q Q}^{1 / q Q}\left(\sum_{M \leq m \leq N} e(m \lambda) m^{-1 / 2}\right)^{2}\left(\sum_{M \leq m \leq N} e(m \lambda)\right) e(-n \lambda) d \lambda \\
& +O\left(\sum_{q \leq P} \frac{1}{\phi^{3}(q)} \phi(q) \cdot \phi^{2}(q) \int_{-1 / q Q}^{1 / q Q}(1+\lambda N)^{2} \min (N, 1 / \lambda) d \lambda\right) \\
= & \frac{1}{4} \sum_{q \leq P} \frac{\mu(q)}{\phi^{3}(q)} \sum_{a=1,(a, q)=1}^{q} e\left(-\frac{a n}{q}\right) C^{2}(a, q) \\
& \times\left(\int_{-1 / 2}^{1 / 2}\left(\sum_{M \leq m \leq N} e(m \lambda) m^{-1 / 2}\right)^{2}\left(\sum_{M \leq m \leq N} e(m \lambda)\right) e(-n \lambda) d \lambda\right. \\
& \left.-2 \int_{1 / q Q}^{1 / 2}\left(\sum_{M \leq m \leq N} e(m \lambda) m^{-1 / 2}\right)^{2}\left(\sum_{M \leq m \leq N} e(m \lambda)\right) e(-n \lambda) d \lambda\right) \\
+ & O\left(N^{2} / Q^{2}\right) .
\end{aligned}
$$

By Vinogradov's upper bound (see [8, Ch. VI, Problem 14b( $\alpha)]$ )

$$
|\mathrm{C}(\chi, a)| \leq 2 q^{1 / 2} d(q)
$$

and $M^{-1} P^{2} Q^{2}=N L^{-14}$, the second sum in $(*)$ is, according to the earlier estimate for $V_{1}(\lambda)$ and $V_{2}(\lambda)$,

$$
\begin{aligned}
& \ll \sum_{q \leq P} \frac{\mu^{2}(q)}{\phi^{3}(q)} \sum_{\substack{a \leq q \\
(a, q)=1}}\left|C^{2}(a, q)\right| \int_{1 / q Q}^{1 / 2} M^{-1} \lambda^{-3} d \lambda \\
& \ll \sum_{q \leq P} \frac{\mu^{2}(q)}{\phi^{2}(q)} q d^{2}(q) M^{-1} P^{2} Q^{2} \ll N L^{-1} .
\end{aligned}
$$

Hence

$$
\begin{aligned}
I_{1}= & \frac{1}{4} \sum_{q \leq P} \frac{\mu(q)}{\phi^{3}(q)} \sum_{\substack{a \leq q \\
(a, q)=1}} e\left(-\frac{a n}{q}\right) C^{2}(a, q) \\
& \times \sum_{M<m_{1} \leq N} \sum_{M<m_{2}<n-M-m_{1}}\left(m_{1} m_{2}\right)^{-1 / 2}+O\left(N L^{-1}\right) \\
= & \frac{1}{4} \pi \sigma_{1}(n) n+O\left(N L^{-1}\right),
\end{aligned}
$$

where $\sigma_{1}(n)$ is defined in (2.1). Therefore,

$$
\int_{\mathbf{M}} S^{2}(\alpha) T(\alpha) e(-n \alpha) d \alpha=\frac{1}{4} \pi \sigma_{1}(n) n+O\left(N L^{-1}\right) .
$$


In the following, we want to show that $\int_{\mathbf{M}} S_{1}^{2}(\alpha) T_{1}(\alpha) e(-n \alpha) d \alpha$ can be replaced by $\int_{\mathbf{M}} S^{2}(\alpha) T(\alpha) e(-n \alpha) d \alpha$. Indeed,

$$
\begin{aligned}
& \int_{\mathbf{M}}\left(S^{2}(\alpha) T(\alpha)-S_{1}^{2}(\alpha) T_{1}(\alpha)\right) e(-n \alpha) d \alpha \\
& \quad \ll \int_{\mathbf{M}}\left|S_{1}^{2}(\alpha)\right|\left|T(\alpha)-T_{1}(\alpha)\right| d \alpha+\int_{0}^{1}\left|S^{2}(\alpha)-S_{1}^{2}(\alpha)\right||T(\alpha)| d \alpha \\
& =: H_{1}+H_{2} .
\end{aligned}
$$

From Cauchy's inequality, we have

$$
H_{1} \leq\left(\int_{0}^{1}\left|\sum_{p \leq M} \log p e(\alpha p)\right|^{2} d \alpha\right)^{1 / 2}\left(\int_{0}^{1}\left|S_{1}(\alpha)\right|^{4} d \alpha\right)^{1 / 2}=: H_{11}^{1 / 2} H_{12}^{1 / 2},
$$

where

$$
\begin{aligned}
& H_{11}=\int_{0}^{1}\left|\sum_{p \leq M} \log p e(\alpha p)\right|^{2} d \alpha \ll L^{2} M, \\
& H_{12}=\int_{0}^{1}\left|S_{1}(\alpha)\right|^{4} d \alpha \ll L^{4} Z(N),
\end{aligned}
$$

where $Z(N)$ is the number of solutions of the equation

$$
p_{1}^{2}+p_{2}^{2}=p_{3}^{2}+p_{4}^{2}
$$

and $p_{j} \leq N, 1 \leq j \leq 4$, are all primes. From [7, Satz 3], the number of $p_{1}, p_{2}, p_{3}, p_{4}$ satisfying $(2.6)$ with $p_{1} p_{2} \neq p_{3} p_{4}$ is $O\left(N L^{-3}\right)$. From the prime number theorem, (2.6) has $O\left(N L^{-2}\right)$ trivial solutions. Hence

$$
H_{12} \leq N L^{2} \text {. }
$$

Therefore

$$
H_{1} \leq\left(M L^{2}\right)^{1 / 2}\left(N L^{2}\right)^{1 / 2} \ll N L^{-5} .
$$

We have

$$
\begin{aligned}
H_{2} \leq & \left(\int_{0}^{1}\left|S(\alpha)-S_{1}(\alpha)\right|^{4} d \alpha\right)^{1 / 4}\left(\int_{0}^{1}\left|S(\alpha)+S_{1}(\alpha)\right|^{4} d \alpha\right)^{1 / 4} \\
& \times\left(\int_{0}^{1}|T(\alpha)|^{2} d \alpha\right)^{1 / 2} \\
= & : H_{21}^{1 / 2} H_{22}^{1 / 4} H_{23}^{1 / 4}
\end{aligned}
$$




$$
\begin{aligned}
H_{21} & =\int_{0}^{1}|T(\alpha)|^{2} d \alpha \ll L^{2} N, \\
H_{22} & =\int_{0}^{1}\left|S(\alpha)-S_{1}(\alpha)\right|^{4} d \alpha \ll L^{4} Z(M),
\end{aligned}
$$

where $Z(M)$ is defined in (2.6). From (2.7) we have

$$
\begin{gathered}
H_{22} \ll M L^{2}=N L^{-12}, \\
H_{23}=\int_{0}^{1}\left|S(\alpha)+S_{1}(\alpha)\right|^{4} d \alpha \ll \int_{0}^{1}|S(\alpha)|^{4} d \alpha+\int_{0}^{1}\left|S_{1}(\alpha)\right|^{4} d \alpha .
\end{gathered}
$$

Since

$$
\begin{aligned}
\int_{0}^{1}|S(\alpha)|^{4} d \alpha & =\sum_{\substack{p_{1}^{2}+p_{2}^{2}=p_{3}^{2}+p_{4}^{2} \\
M<p_{i}^{2} \leq N}} \log p_{1} \cdots \log p_{4} \leq \sum_{\substack{p_{1}^{2}+p_{2}^{2}=p_{3}^{2}+p_{4}^{2} \\
p_{i}^{2} \leq N}} \log p_{1} \cdots \log p_{4} \\
& =\int_{0}^{1}\left|S_{1}(\alpha)\right|^{4} d \alpha,
\end{aligned}
$$

we have

$$
H_{23} \ll \int_{0}^{1}\left|S_{1}(\alpha)\right|^{4} d \alpha=\sum_{\substack{p_{1}^{2}+p_{2}^{2}=p_{3}^{2}+p_{4}^{2} \\ p_{i}^{2} \leq N}} \log p_{1} \cdots \log p_{4} \ll L^{4} Z(N) \ll N L^{2} .
$$

Hence

$$
\begin{gathered}
H_{2} \leq H_{21}^{1 / 2} H_{22}^{1 / 4} H_{23}^{1 / 4} \ll\left(N L^{2}\right)^{1 / 2}\left(N L^{-12}\right)^{1 / 4}\left(N L^{2}\right)^{1 / 4} \ll N L^{-1}, \\
\int_{\mathbf{M}}\left(S^{2}(\alpha) T(\alpha)-S_{1}^{2}(\alpha) T_{1}(\alpha)\right) e(-n \alpha) d \alpha=O\left(N L^{-1}\right) .
\end{gathered}
$$

Therefore we have

$$
\int_{\mathbf{M}} S_{1}^{2}(\alpha) T_{1}(\alpha) e(-n \alpha) d \alpha=\int_{\mathbf{M}} S^{2}(\alpha) T(\alpha) e(-n \alpha) d \alpha+O\left(N L^{-1}\right) .
$$

Let

$$
\begin{gathered}
\Xi(N, k)=\left\{n: n=N-\left(2^{\nu_{1}}+\cdots+2^{\nu_{k}}\right)\right\}, \\
A_{1}=\left\{n=N-2^{\nu_{1}}-2^{\nu_{2}}-\cdots-2^{\nu_{k}}: \nu_{i} \leq \log _{2}(N / k L), 1 \leq i \leq k\right\} .
\end{gathered}
$$

We have

$$
\sum_{n \in \Xi(N, k)} \int_{\mathbf{M}} T_{1}(\alpha) S_{1}^{2}(\alpha) e(-n \alpha) d \alpha=\frac{\pi}{4} \sum_{n \in \Xi(N, k)} \sigma_{1}(n) n+O\left(N L^{k-1}\right) .
$$


Therefore

$$
\begin{aligned}
& \sum_{n \in \Xi(N, k) \mathbf{M}} \int_{1} T_{1}(\alpha) S_{1}^{2}(\alpha) e(-n \alpha) d \alpha \\
&=\frac{\pi}{4} \sum_{n \in \Xi(N, k)} \sigma_{1}(n) n+O\left(N L^{k-1}\right) \\
& \geq \frac{\pi}{4} \sum_{n \in A_{1}} \sigma_{1}(n) n+O\left(N L^{k-1}\right) \\
&=\frac{\pi}{4} \sum_{n \in A_{1}} \sigma_{1}(n)\left(N-2^{\nu_{1}}-\cdots-2^{\nu_{k}}\right)+O\left(N L^{k-1}\right) \\
& \geq \frac{\pi}{4} N\left(1-L^{-1}\right) \sum_{n \in A_{1}} \sigma_{1}(n)+O\left(N L^{k-1}\right) \\
& \geq \frac{\pi}{4} N(1-\delta) \sum_{n \in A_{1}} \sigma_{1}(n)+O\left(N L^{k-1}\right),
\end{aligned}
$$

where $\delta$ is a sufficiently small positive number. Now, we will mainly deal with $\sigma_{1}(n)$.

3. The singular series. We need the following lemmas:

Lemma 3.1 (see [5, Lemma 3]). Let $\eta<1 / 7$ e. The set $\mathcal{E}$ of $\alpha \in[0,1]$ for which $|G(\alpha)| \geq(1-\eta) L$ has measure $\leq L^{5 / 2} N^{\Theta-1}$, where

$$
\begin{aligned}
\Theta=\Theta(\eta)= & \frac{1}{\log 2} \eta \csc ^{2}\left(\frac{\pi}{8}\right) \log \left(\frac{1}{\eta \csc ^{2}(\pi / 8)}\right) \\
& +\frac{1}{\log 2}\left(1-\eta \csc ^{2}\left(\frac{\pi}{8}\right)\right) \log \left(\frac{1}{1-\eta \csc (\pi / 8)}\right) .
\end{aligned}
$$

Lemma 3.2 (see [5, Lemma 4]). If $\alpha$ is a rational number with odd denominator $q$ satisfying $1<\xi(q)<L$, then

$$
|G(\alpha)| \leq\left(1-\frac{1}{\xi(q) \csc ^{2}(\pi / 8)}+\frac{2}{L}\right) L,
$$

where $\xi(q)$ denotes the least positive integer $\xi$ satisfying

$$
2^{\xi} \equiv 1(\bmod q)
$$

for odd $q$.

Lemma 3.3. Let $A(q)=\prod_{p \mid q} A(p)$, where $p$ is prime and $A(p)$ satisfies

$$
A(p)= \begin{cases}\sqrt{p}+1 & \text { if } p \equiv 1(\bmod 4) \\ \sqrt{p+1} & \text { if } p \equiv-1(\bmod 4) .\end{cases}
$$


Then

$$
\sum_{\xi(q) \leq x} \frac{\mu^{2}(q)}{\phi^{3}(q)} A^{2}(q) q \leq c_{1} \log ^{2} x
$$

and

$$
\sum_{\xi(q) \leq x} \frac{\mu^{2}(q)}{\phi^{2}(q)} A^{2}(q) \leq c_{2} \log ^{1.5} x,
$$

where $c_{1}=5.287076611, c_{2}=3.803$.

Proof. Let

$$
X=\prod_{\xi \leq x}\left(2^{\xi}-1\right)
$$

If $\xi(q) \leq x$, then $q \mid X$, and obviously $2 \nmid X, X \leq 2^{x^{2}}$. We have

$$
\sum_{\xi(q) \leq x} \frac{\mu^{2}(q)}{\phi^{3}(q)} A^{2}(q) q \leq \sum_{q \mid X} \frac{\mu^{2}(q)}{\phi^{3}(q)} A^{2}(q) q \leq \prod_{p \mid X}\left(1+\frac{A^{2}(p)}{\phi^{3}(p)} p\right) .
$$

When $p \geq 16$,

$$
1+\frac{A^{2}(p)}{(p-1)^{3}} p<1+\frac{2}{p-1} .
$$

By Lemma 3.3, we have

$$
\begin{aligned}
\prod_{3 \leq p \mid X}\left(1+\frac{A^{2}(p) p}{(p-1)^{3}}\right) & \leq \frac{5}{4} \prod_{3 \leq p \mid X}\left(1+\frac{2}{p-1}\right) \\
& =\prod_{p \mid 2 X}\left(1+\frac{2}{p-1}\right) \cdot \frac{5}{12} \leq \prod_{p \mid 2 X}\left(1+\frac{1}{p-1}\right)^{2} \cdot \frac{5}{12} \\
& =\frac{(2 X)^{2}}{\phi^{2}(2 X)} \cdot \frac{5}{12} \leq \frac{5}{12} \cdot 4 e^{2 \gamma} \log ^{2} x=: c_{1} \log ^{2} x,
\end{aligned}
$$

since by Lemma 5 of [5], we have

$$
\frac{2 X}{\phi(2 X)}<2 e^{\gamma} \log x
$$

Note that

$$
1.7810<e^{\gamma}<1.78108
$$

So we have

$$
\sum_{\xi(q) \leq x} \frac{\mu^{2}(q) A^{2}(q) q}{\phi^{3}(q)}<c_{1} \log ^{2} x
$$

where

$$
c_{1}=5.287076611 \text {. }
$$


We have

$$
\sum_{\xi(q) \leq n} \frac{\mu^{2}(q)}{\phi^{2}(q)} A^{2}(q) \leq \sum_{q \mid X} \frac{\mu^{2}(q)}{\phi^{2}(q)} A^{2}(q)=\prod_{p \mid X}\left(1+\frac{A^{2}(p)}{\phi^{2}(p)}\right),
$$

and for $p \geq 25$,

$$
1+\frac{A^{2}(p)}{(p-1)^{2}} \leq 1+\frac{1.5}{p-1}
$$

Thus

$$
\begin{aligned}
\prod_{3 \leq p \mid X}\left(1+\frac{A^{2}(p)}{\phi^{2}(p)}\right) & \leq \prod_{3 \leq p \mid X}\left(1+\frac{1.5}{p-1}\right) \cdot 1.413867968 \\
& =\prod_{p \mid 2 X}\left(1+\frac{1.5}{p-1}\right) \cdot \frac{1}{2.5} \cdot 1.413867968 \\
& \leq \prod_{p \mid 2 X}\left(1+\frac{1}{p-1}\right)^{1.5} \cdot \frac{1}{2.5} \cdot 1.413867968 \\
& <3.803 \log ^{1.5} x
\end{aligned}
$$

where we have used (3.1) and (3.2).

Lemma 3.4 (see [5, Lemma 6]). For odd $q$ and $k \geq 2$, we have

$$
r_{k, k}(0) \leq 2 L^{2 k-2}, \quad \sum_{q \mid n} r_{k, k}(n) \leq L^{2 k-1}\left(1+\frac{L}{\xi(q)}\right),
$$

where $r_{k, k}(n)$ is the number of ways to write $n$ as

$$
n=2^{\nu_{1}}+\cdots+2^{\nu_{k}}-2^{\mu_{1}}-\cdots-2^{\mu_{k}} \quad\left(1 \leq \nu_{i}, \mu_{i} \leq L\right) .
$$

Consider the sum

$$
\sum=\sum_{\substack{3 \leq q \leq R \\ 2 \nmid q}} \frac{\mu^{2}(q)}{\phi^{3}(q)} \sum_{\substack{a=1 \\(a, q)=1}}^{q}\left|C^{2}(a, q)\right|\left|G^{k}(a / q)\right| .
$$

According to the length of $\xi(q)$, we obtain

$$
\sum=\left(\sum_{\substack{3 \leq q \leq R \\ \xi(q) \leq E}}+\sum_{\substack{3 \leq q \leq R \\ \xi(q)>E}}\right) \frac{\mu^{2}(q)}{\phi^{3}(q)} \sum_{\substack{a=1 \\(a, q)=1}}^{q}\left|C^{2}(a, q)\right|\left|G^{k}(a / q)\right|,
$$

where $E$ is a constant $\leq L$. 
By Lemmas 3.2 and 3.3, the first sum on the right hand side of (3.3) is

$$
\begin{aligned}
\sum_{\substack{3 \leq q \leq R \\
\xi(q) \leq E}} \leq L^{k}\left(1-\frac{1}{E \csc ^{2}(\pi / 8)}\right)^{k} \sum_{\substack{3 \leq q \leq R \\
\xi(q) \leq E}} \frac{\mu^{2}(q)}{\phi^{2}(q)} A^{2}(q) \\
\leq c_{2} \log ^{1.5} E\left(1-\frac{1}{E \csc ^{2}(\pi / 8)}\right)^{k} L^{k} .
\end{aligned}
$$

Remark. $C(a, q)$ is multiplicative, so for $q=p_{1} p_{2} \cdots p_{s}, C(a, q)=$ $C\left(a_{1}, p_{1}\right) \cdots C\left(a_{s}, p_{s}\right)$, with $a_{i}=a q / p_{i}$. It is easy to see that when $p_{i} \equiv 1$ $(\bmod 4)$, we have $\left|C\left(a_{i}, p_{i}\right)\right|=\left| \pm \sqrt{p_{i}}-1\right| \leq \sqrt{p_{i}}+1$, while when $p_{i} \equiv-1$ $(\bmod 4),\left|C\left(a_{i}, p_{i}\right)\right|=\left|\sqrt{-p_{i}}-1\right| \leq \sqrt{p_{i}}+1$. Thus we have

$$
\left|C\left(a_{i}, p_{i}\right)\right| \leq A\left(p_{i}\right), \quad|C(a, q)| \leq \prod_{p \mid q} A(p)=A(q) .
$$

For the last sum in (3.3), we use Lemma 3.4. When $k=2 m$,

$$
\begin{aligned}
\sum_{\substack{a=1 \\
(a, q)=1}}^{q}|G(a / q)|^{k} & =\sum_{\substack{a=1 \\
(a, q)=1}}^{q}|G(a / q)|^{2 m} \leq \sum_{a=1}^{q}|G(a / q)|^{2 m}=q \sum_{q \mid n} r_{m, m}(n) \\
& \leq q L^{2 m-1}\left(1+\frac{L}{\xi(q)}\right)=q L^{k-1}+\frac{q L^{k}}{\xi(q)} .
\end{aligned}
$$

When $k=2 m+1$,

$$
\begin{aligned}
\sum_{\substack{a=1 \\
(a, q)=1}}^{q}|G(a / q)|^{k} & =\sum_{\substack{a=1 \\
(a, q)=1}}^{q}|G(a / q)|^{2 m+1} \leq L \sum_{\substack{a=1 \\
(a, q)=1}}^{q}|G(a / q)|^{2 m} \\
& \leq L\left(q L^{2 m-1}\left(1+\frac{L}{\xi(q)}\right)\right)=q L^{k-1}+\frac{q L^{k}}{\xi(q)} .
\end{aligned}
$$

Thus we have

$$
\sum_{\substack{a=1 \\(a, q)=1}}^{q}|G(a / q)|^{k} \leq q L^{k-1}+\frac{q L^{k}}{\xi(q)},
$$

and so

$$
\begin{aligned}
\sum_{\substack{3 \leq q \leq R \\
\xi(q)>E}} \leq \sum_{\substack{3 \leq q \leq R \\
\xi(q)>E}} \frac{\mu^{2}(q)}{\phi^{3}(q)} \sum_{\substack{a=1 \\
(a, q)=1}}^{q}\left|C^{2}(a, q)\right|\left|G^{k}(a / q)\right| \\
\leq \sum_{\substack{3 \leq q \leq R \\
\xi(q)>E}} \frac{\mu^{2}(q)}{\phi^{3}(q)} A^{2}(q) \sum_{\substack{a=1 \\
(a, q)=1}}^{q}\left|G^{k}(a / q)\right|
\end{aligned}
$$




$$
\leq L^{k-1} \sum_{3 \leq q \leq R} \frac{\mu^{2}(q)}{\phi^{3}(q)} A^{2}(q) q+L^{k} \sum_{\substack{3 \leq q \leq R \\ \xi(q)>E}} \frac{\mu^{2}(q)}{\phi^{3}(q)} A^{2}(q) \frac{q}{\xi(q)} .
$$

By Lemma 3.3, the first sum on the right hand side above is $\ll \log ^{2} R$. The second sum is

$$
\begin{aligned}
& \leq \sum_{m>E} \frac{1}{m} \sum_{\xi(q)=m} \frac{\mu^{2}(q)}{\phi^{3}(q)} A^{2}(q) q \leq \int_{E}^{\infty} \frac{1}{t^{2}}\left(\sum_{\xi(q) \leq t} \frac{\mu^{2}(q)}{\phi^{3}(q)} A^{2}(q) q\right) d t \\
& \leq c_{1} \int_{E}^{\infty} \frac{\log ^{2} t}{t^{2}} d t=c_{1}\left(\frac{\log ^{2} E}{E}+\frac{2 \log E}{E}+\frac{2}{E}\right) .
\end{aligned}
$$

Hence

$$
\sum_{\substack{3 \leq q \leq R \\ \xi(q)>E}} \leq c_{1} L^{k}\left(\frac{\log ^{2} E}{E}+\frac{2 \log E}{E}+\frac{2}{E}\right)+O\left(L^{k-1} \log ^{2} R\right) .
$$

Therefore

$$
\begin{aligned}
\sum \leq & c_{1} L^{k}\left(\frac{\log ^{2} E}{E}+\frac{2 \log E}{E}+\frac{2}{E}\right) \\
& +c_{2} \log ^{1.5} E\left(1-\frac{1}{E \csc ^{2}(\pi / 8)}\right)^{k} L^{k}+O\left(L^{k-1} \log ^{2} R\right)
\end{aligned}
$$

Define

$$
\begin{gathered}
A(n, q):=\frac{\mu(q)}{\phi^{3}(q)} \sum_{\substack{a=1 \\
(a, q)=1}}^{q} C^{2}(a, q) e\left(-\frac{a n}{q}\right), \\
\sigma_{1}(n)=\sum_{q \leq P} A(n, q), \quad \sigma_{0}(n)=\sum_{q=1}^{\infty} A(n, q), \quad \sigma(n)=\sum_{q \leq R} A(n, q) .
\end{gathered}
$$

We will prove that

$$
|A(n, p)| \leq \begin{cases}\frac{3 p+1}{(p-1)^{3}} & \text { if } p \nmid n, \\ \frac{p^{2}-1}{(p-1)^{3}} & \text { if } p \mid n .\end{cases}
$$

We have

$$
C(a, p)=\chi(a) S(p, 1)-1
$$

where $(a, p)=1, \chi(a)=\left(\frac{a}{p}\right)$ is the Legendre symbol and

$$
S(p, 1)=\sum_{h=1}^{p} e\left(\frac{h^{2}}{p}\right)
$$


is the Gauss sum. Here $S(p, 1)$ satisfies

$$
S(p, 1)= \begin{cases}\sqrt{p} & \text { if } p \equiv 1(\bmod 4), \\ i \sqrt{p} & \text { if } p \equiv-1(\bmod 4) .\end{cases}
$$

Therefore, we have

$$
\begin{aligned}
\sum_{a=1}^{p-1} C^{2} & (a, p) e\left(-\frac{a n}{p}\right)=\sum_{a=1}^{p-1}(\chi(a) S(p, 1)-1)^{2} e\left(-\frac{a n}{p}\right) \\
= & \left(S^{2}(p, 1)+1\right) \sum_{a=1}^{p-1} e\left(-\frac{a n}{p}\right)-2 S(p, 1) \sum_{a=1}^{p-1} \chi(a) e\left(-\frac{a n}{p}\right) \\
= & : \mathbf{S}_{1}-\mathbf{S}_{2} .
\end{aligned}
$$

For $\mathbf{S}_{1}$, we need the following result:

$$
\sum_{a=1}^{p-1} e\left(-\frac{a n}{p}\right)= \begin{cases}p-1 & \text { if } p \mid n, \\ -1 & \text { if } p \nmid n .\end{cases}
$$

By this result and (3.5), we obtain

$$
\mathbf{S}_{1}= \begin{cases}p^{2}-1, & p \equiv 1(\bmod 4), p \mid n, \\ -(p-1)^{2}, & p \equiv-1(\bmod 4), p \mid n, \\ -(p+1), & p \equiv 1(\bmod 4), p \nmid n, \\ p-1, & p \equiv-1(\bmod 4), p \nmid n .\end{cases}
$$

For $\mathbf{S}_{2}$, if $p \mid n$, then $\sum_{a=1}^{p-1} \chi(a)=0$; if $p \nmid n$, let

$$
F(n)=\sum_{a=1}^{p}\left(\frac{a}{p}\right) e\left(\frac{a n}{p}\right) .
$$

Then obviously we have

$$
F(n)=\left(\frac{n}{p}\right) F(1)
$$

and

$$
\begin{aligned}
S(p, 1) & =\sum_{m=1}^{p} e\left(\frac{m^{2}}{p}\right)=\sum_{m=1}^{p-1} e\left(\frac{m^{2}}{p}\right)+1 \\
& =\sum_{a=1}^{p-1}\left(1+\left(\frac{a}{p}\right)\right) e\left(\frac{a}{p}\right)+1=\sum_{a=1}^{p}\left(\frac{a}{p}\right) e\left(\frac{a}{p}\right)=F(1) .
\end{aligned}
$$

Hence

$$
\mathbf{S}_{2}=2 S^{2}(p, 1)\left(-\frac{n}{p}\right) .
$$


From (3.5), (3.6) and (3.8), we get

$$
\begin{aligned}
\sum_{a=1}^{p-1} C^{2}(a, p) e\left(-\frac{a n}{p}\right) & \\
= & \begin{cases}\mathbf{S}_{1}, & p \mid n, \\
-(p+1)-2 p=-3 p-1, & \left(-\frac{n}{p}\right)=1, p \equiv 1(\bmod 4), \\
(p-1)+2 p=3 p-1, & \left(-\frac{n}{p}\right)=1, p \equiv-1(\bmod 4), \\
-(p+1)+2 p=p-1, & \left(-\frac{n}{p}\right)=-1, p \equiv 1(\bmod 4), \\
(p-1)-2 p=-(p+1), & \left(-\frac{n}{p}\right)=-1, p \equiv-1(\bmod 4) .\end{cases}
\end{aligned}
$$

Therefore, we have

$$
|A(n, p)| \leq \begin{cases}\frac{3 p+1}{(p-1)^{3}} & \text { if } p \nmid n, \\ \frac{p^{2}-1}{(p-1)^{3}} & \text { if } p \mid n .\end{cases}
$$

Thus

$$
|A(n, q)| \leq 2 \prod_{\substack{p \mid q \\ p \nmid n}} \frac{25}{p^{2}} \prod_{\substack{p|q \\ p| n}} \frac{25}{p}=2 \prod_{p \mid q} \frac{25}{p^{2}} \prod_{\substack{p|q \\ p| n}} p \ll q^{-2+\varepsilon}(q, n) .
$$

Therefore

$$
\sum_{q>x}|A(n, q)| \ll \sum_{s \mid n} s \sum_{s t>x}(s t)^{-2+\varepsilon} \ll x^{-1+\varepsilon} d(n) .
$$

Thus,

$$
\sigma_{1}(n)=\sigma_{0}(n)+O\left(P^{-1+\varepsilon}\right), \quad \sigma_{1}(n)=\sigma(n)+O\left(R^{-1+\varepsilon}\right) .
$$

Obviously, the function of $q$

$$
\frac{\mu(q)}{\phi^{3}(q)} \sum_{\substack{a=1 \\(a, q)=1}}^{q} C^{2}(a, q) e\left(-\frac{a n}{q}\right)
$$

is multiplicative, therefore we have

$$
\begin{aligned}
\sigma_{0}(n) & =\sum_{q=1}^{\infty} \frac{\mu(q)}{\phi^{3}(q)} \sum_{\substack{a=1 \\
(a, q)=1}}^{q} C^{2}(a, q) e\left(-\frac{a n}{q}\right) \\
& =\prod_{p}\left(1+\frac{1}{\phi^{3}(p)} \sum_{a=1}^{p-1} C^{2}(a, p) e\left(-\frac{a n}{p}\right)\right) .
\end{aligned}
$$


This infinite series is positive and has positive partial sums, it must converge to a positive constant, say $c$, i.e.

$$
\sigma_{0}(n)=\prod_{p}(1+A(n, p))=c>0 .
$$

When $n$ is sufficiently large, we have $\sigma_{1}(n)>0, \sigma(n)>0$. Thus, we could replace $\sigma_{1}(n)$ by $\sigma(n)$. Take

$$
\varepsilon=\frac{\log \log \log R}{\log N}, \quad R=o(N) .
$$

We have

$$
\sum_{n \in A_{1}} \sigma_{1}(n)=\sum_{n \in A_{1}} \sigma(n)+O\left(L^{k} R^{-1+\varepsilon}\right)
$$

Define

$$
\begin{aligned}
\sum_{n \in A_{1}} \sigma(n) & =\sum_{q \leq R} \frac{\mu^{2}(q)}{\phi^{3}(q)} \sum_{\substack{a=1 \\
(a, q)=1}}^{q} C^{2}(a, q) e\left(-\frac{a N}{q}\right)\left(\sum_{\nu=1}^{\log _{2}(N / k L)} e\left(\frac{a}{q} 2^{\nu}\right)\right)^{k} \\
& =: \sum_{q \leq R} F(q, N) .
\end{aligned}
$$

When $q=1,2, F(q, N)$ contributes $2 L^{k}$ to the above sum. Thus,

$$
\begin{aligned}
\sum_{n \in A_{1}} \sigma(n) & =2 L^{k}+\sum_{\substack{3 \leq q \leq R\\
}} F(q, N) \\
& =2 L^{k}+\sum_{\substack{3 \leq q \leq R \\
2 \nmid q}} F(q, N)+\sum_{\substack{3 \leq q \leq R / 2 \\
2 \nmid q}} F(q, N) \\
& =: 2 L^{k}+\sum_{1}+\sum_{2} .
\end{aligned}
$$

From (3.4), we have

$$
\begin{aligned}
\left|\sum_{1}+\sum_{2}\right| \leq & 2 c_{1} L^{k}\left(\frac{\log ^{2} E}{E}+\frac{2 \log E}{E}+\frac{2}{E}\right) \\
& +2 c_{2} \log ^{1.5} E\left(1-\frac{1}{E \csc ^{2}(\pi / 8)}\right)^{k} L^{k} \\
& +O\left(L^{k-1} \log ^{2} R\right),
\end{aligned}
$$

where $c_{1}=5.287076611, c_{2}=3.803$. If we take $R=\exp (\sqrt{\log N} / \log \log N)$, the $O$-term above is $O\left(L^{k}(\log \log N)^{-2}\right)$. 


\section{The proof of Theorem 1}

Lemma 4.1 (see [3, Lemma 5.1]). For $p$ a prime,

$$
\begin{gathered}
T(\alpha)=\sum_{p \leq N} \log p e(\alpha p), \quad S(\alpha)=\sum_{p^{2} \leq N} \log p e\left(\alpha p^{2}\right), \\
G(\alpha)=\sum_{\nu \leq \log _{2} N} e\left(\alpha 2^{\nu}\right),
\end{gathered}
$$

we have

$$
\int_{0}^{1}|T(\alpha) G(\alpha)|^{4} d \alpha \leq c_{5} \frac{\pi^{2}}{16} N L^{4}
$$

where

$$
c_{5} \leq\left(\frac{11^{4} \cdot 43 \cdot \pi^{24}}{2^{24} \cdot 5^{2}}+\frac{8}{\pi^{2}} \log ^{2} 2\right)(1+\varepsilon)^{9} .
$$

Lemma 4.2 (see [5, Lemma 9]). We have

$$
\int_{0}^{1}|S(\alpha) G(\alpha)|^{2} d \alpha \leq \frac{2}{\log ^{2} 2} c_{3} N L^{2},
$$

where $c_{3}<17.2435$.

REMARK. It follows from the proofs of the above lemmas that they remain true if $T(\alpha)$ and $S(\alpha)$ are replaced by $T_{1}(\alpha)$ and $S_{1}(\alpha)$ respectively.

From (2.8), (3.9) and (3.10), we have

$$
\int_{\mathbf{M}} \geq\left(\frac{\pi}{2}-\delta\right) N L^{k}+\left(\sum_{1}+\sum_{2}\right),
$$

where

$$
\begin{aligned}
\left|\sum_{1}+\sum_{2}\right| \leq & 2 c_{1} N L^{k}\left(\frac{\log ^{2} E}{E}+2 \frac{\log E}{E}+\frac{2}{E}\right) \\
& +2 c_{2} \log ^{1.5} E\left(1-\frac{1}{E \csc ^{2}(\pi / 8)}\right)^{k} .
\end{aligned}
$$

Let

$$
r_{k}(N)=\int_{0}^{1} T_{1}(\alpha) S_{1}^{2}(\alpha) G^{k}(\alpha) e(-N \alpha) d \alpha=\int_{\mathbf{M}}+\int_{C(\mathbf{M}) \cap \mathcal{E}}+\int_{C(\mathbf{M}) \cap C(\mathcal{E})}
$$

with the set $\mathcal{E}$ from Lemma 3.1.

In order to estimate the minor arcs, we first estimate the second integral. For $\alpha \in C(\mathbf{M}), \alpha=a / q+\lambda, 1 \leq a \leq q,(a, q)=1$, where $P \leq q \leq Q$, we use Theorem 3 of [1] to obtain

$$
S_{1}(\alpha) \ll N^{1 / 2+\varepsilon}\left(P^{-1}+N^{-1 / 4}+Q N^{-1}\right)^{1 / 4} \ll N^{1 / 2-1 / 30} .
$$


Take $\eta=1 / 868$, so $\Theta$ in Lemma 3.1 is $<1 / 15$. Thus the second integral can be estimated as

$$
\int_{C(\mathbf{M}) \cap \mathcal{E}} \leq N^{\Theta-1} N^{1-1 / 15+4 \varepsilon} L^{k} N \ll N L^{-1+k} .
$$

The last integral can be estimated as

$$
\begin{aligned}
& \int_{C(\mathbf{M}) \cap C(\mathcal{E})} \\
& \leq((1-\eta) L)^{k-3}\left(\int_{0}^{1}\left|T_{1}(\alpha) G(\alpha)\right|^{2} d \alpha\right)^{1 / 2}\left(\int_{0}^{1}\left|S_{1}(\alpha) G(\alpha)\right|^{4} d \alpha\right)^{1 / 2} .
\end{aligned}
$$

From (4.4), Lemma 4.1 and Lemma 4.2, we have

$$
\int_{C(\mathbf{M}) \cap C(\mathcal{E})} \leq 23667416(1-\eta)^{k-3}\left(N L^{k}\right) .
$$

Take $E=400$; when $k \geq 22000$, from (4.1)-(4.3) and (4.5), we have

$$
\begin{aligned}
r_{k}(N) \geq & \left(\frac{\pi}{2}-2 \delta\right) N L^{k}-23667416(1-\eta)^{k-3} N L^{k} \\
& -N L^{k}\left(2 c_{1} N L^{k}\left(\frac{\log ^{2} E}{E}+2 \frac{\log E}{E}+\frac{2}{E}\right)\right. \\
& \left.+2 c_{2} \log ^{1.5} E\left(1-\frac{1}{E \csc ^{2}(\pi / 8)}\right)^{k}\right)>0
\end{aligned}
$$

Thus the proof of Theorem 1 is complete.

Acknowledgements. The author is grateful to Professor Liu Jianya, Professor Kikumasa, the referee and the copy editor for their helpful comments concerning this work.

\section{References}

[1] A. Ghosh, The distribution of $\alpha p^{2}$ modulo 1, Proc. London Math. Soc. (3) 42 (1981), 252-269.

[2] M. C. Leung and M. C. Liu, On generalized quadratic equations in three prime variables, Monatsh. Math. 115 (1993), 133-169.

[3] J. Y. Liu and M. C. Liu, Representation of even integers as sums of squares of primes and powers of 2, J. Number Theory 83 (2000), 202-225.

[4] - - - The exceptional set in the four prime squares problem, Illinois J. Math. 44 (2000), 272-293.

[5] J. Y. Liu, M. C. Liu and T. Z. Wang, The number of powers of 2 in a representation of large even integers (I), Sci. China 41 (1998), 386-398.

[6] J. Y. Liu, M. C. Liu and T. Zhan, Squares of primes and powers of two, Monatsh. Math. 128 (1999), 283-313. 
[7] G. J. Rieger, Über die Summe aus einem Quadrat und einem Primzahlquadrat, J. Reine Angew. Math. 251 (1968), 89-100.

[8] I. M. Vinogradov, Elements of Number Theory, Dover, New York, 1954.

Network and Information Security Institute

Shandong University

Jinan, Shandong, China

E-mail: liutao@sdu.edu.cn

Received on 6.5.2002

and in revised form on 12.8.2004 